\title{
Basic science (August 2005)
}

1. Aupperlee MD, Smith KT, Kariagina A, Haslam SZ. Progesterone receptor isoforms $\mathrm{A}$ and $\mathrm{B}$ : temporal and spatial differences in expression during murine mammary gland development. Endocrinology 2005; 146: 3577-3588.

2. Bar-Sinai A, Bassa N, Fischette $M$, Gottesman MM, Love DC, Hanover JA, Hochman J. Mouse mammary tumor virus envderived peptide associates with nucleolar targets in lymphoma, mammary carcinoma, and human breast cancer. Cancer Res 2005; 65: 7223-7230.

3. Berteaux N, Lottin V, Monte D, Pinte S, Quatannens B, Coll J, Hondermarck H, Curgy JJ, Dugimont T, Adriaenssens E. H19 mRNA-like noncoding RNA promotes breast cancer cell proliferation through positive control by E2F1. J Biol Chem 2005; 280: 29625-29636.

4. Biroccio A, D'Angelo C, Jansen B, Cleave ME, Zupi G. Antisense clusterin oligodeoxynucleotides increase the response of HER-2 gene amplified breast cancer cells to trastuzumab. J Cell Physiol 2005; 204: 463-469.

5. Bogdanova N, Enssen-Dubrowinskaja N, Feshchenko S, Lazjuk GI, Rogov YI, Dammann O, Bremer M, Karstens JH, Sohn C, Dork T. Association of two mutations in the CHEK2 gene with breast cancer. Int J Cancer 2005; 116: 263-266.

6. Cabioglu N, Yazici MS, Arun B, Broglio KR, Hortobagyi GN, Price JE, Sahin A. CCR7 and CXCR4 as novel biomarkers predicting axillary lymph node metastasis in T-1 breast cancer. Clin Cancer Res 2005; 11: 5686-5693.

7. Ceschi M, Sun CL, Van Den Berg D, Koh WP, Yu MC, Probst-Hensch N. The effect of cyclin D1 (CCND1) G870A-polymorphism on breast cancer risk is modified by oxidative stress among Chinese women in Singapore. Carcinogenesis 2005; 26: 1457-1464.

8. Chen B, Pan HY, Zhu LY, Deng Y, Pollard JW. Progesterone inhibits the estrogen-induced

First published online 03/01/06 BCO/505/2005/JW phosphoinositide 3-Kinase $\rightarrow$ AKT $\rightarrow$ GSK-beta $3 \rightarrow$ Cyclin D1 $\rightarrow$ pRB pathway to block uterine epithelial cell proliferation. Mol Endocrinol 2005; 19: 1978-1990.

9. Cook AC, Tuck AB, McCarthy S, Turner JG, Irby RB, Bloom GC, Yeatman TJ, Chambers AF. Osteopontin induces multiple changes in gene expression that reflect the six 'hallmarks of cancer' in a model of breast cancer progression. Mol Carcinogen 2005; 43: 225-236.

10. DeMichele A, Aplenc R, Botbyl J, Colligan T, Wray L, Klein-Cabral M, Foulkes A, Gimotty P, Glick J, Weber B, Stadtmauer E, Rebbeck TR. Drug-metabolizing enzyme polymorphisms predict clinical outcome in a node-positive breast cancer cohort. J Clin Oncol 2005; 23: 5552-5559.

11. Dietel M, Lewis MA, Shapiro S. Hormone replacement therapy: pathobiological aspects of hormone-sensitive cancers in women relevant to epidemiological studies on HRT: a minireview. Hum Reprod 2005; 20: 2052-2060.

12. Dote H, Cerna D, Burgan CE, Camphausen K, Tofilon PJ. ErbB3 expression predicts tumor cell radiosensitization induced by Hsp90 inhibition. Cancer Res 2005; 65: 6967-6975.

13. Ewan KBR, Oketch-Rabah HA, Ravani SA, Shyamala G, Moses HL, Barcellos-Hoff MH. Proliferation of estrogen receptor-alpha-positive mammary epithelial cells is restrained by transforming growth factor-beta 1 in adult mice. Am J Pathol 2005; 167: 409-417.

14. Fernandez CA, Yan L, Lous G, Yang J, Kutok JL, Moses MA. The matrix metalloproteinase9/neutrophil gelatinase-associated lipocalin complex plays a role in breast tumor growth and is present in the urine of breast cancer patients. Clin Cancer Res 2005; 11: 5390-5395.

15. Freedman ML, Penney KL, Stram DO, Riley S, McKean-Cowdin R, Le Marchand L, Altshuler D, Haiman CA. A haplotype-based case-control study of BRCA1 and sporadic breast cancer risk. Cancer Res 2005; 65: 7516-7522.

16. Garcia MJ, Pole JCM, Chin SF, Teschendorff A, Naderi A, Ozdag $H$, Vias $M$, Kranjac T, Subkhankulova T, Paish C, Ellis I, Brenton JD, 
Edwards PAW, Caldas C. A $1 \mathrm{Mb}$ minimal amplicon at 8p11-12 in breast cancer identifies new candidate oncogenes. Oncogene 2005; 24: 5235-5245.

17. Hu M, Yao J, Cai L, Bachman KE, van den Brule F, Velculescu V, Polyak K. Distinct epigenetic changes in the stromal cells of breast cancers. Nat Genet 2005; 37: 899-905.

18. Indik S, Gunzburg WH, Salmons B, Rouault F. Mouse mammary tumor virus infects human cells. Cancer Res 2005; 65: 6651-6659.

19. Iorio MV, Ferracin M, Liu CG, Veronese A, Spizzo R, Sabbioni S, Magri E, Pedriali M, Fabbri M, Campiglio M, Menard S, Palazzo JP, Rosenberg A, Musiani P, Volinia S, Nenci I, Calin GA, Querzoli P, Negrini M, Croce CM. MicroRNA gene expression deregulation in human breast cancer. Cancer Res 2005; 65: 7065-7070.

20. Jiang WG, Davies G, Martin T, Parr C, Watkins G, Mason MD, Mokbel K, Mansel RE. Targeting matrilysin and its impact on tumor growth in vivo: the potential implications in breast cancer therapy. Clin Cancer Res 2005; 11: 6012-6019.

21. Karsan A, Eigl BJ, Flibotte S, Gelmon K, Switzer P, Hassell P, Harrison D, Law J, Hayes M, Stillwell M, Xiao Z, Conrads TP, Veenstra T. Analytical and preanalytical biases in serum proteomic pattern analysis for breast cancer diagnosis. Clin Chem 2005; 51: 1525-1528.

22. Keen JC, Zhou Q, Park BH, Pettit C, Mack KM, Blair B, Brenner K, Davidson NE. Protein phosphatase $2 A$ regulates estrogen receptor alpha (ER) expression through modulation of ER mRNA stability. J Biol Chem 2005; 280: 29519-29524.

23. Kim H, Laing M, Muller W. c-Src-null mice exhibit defects in normal mammary gland development and ER alpha signaling. Oncogene 2005; 24: 5629-5636.

24. Koblinski JE, Kaplan-Singer BR, VanOsdol SJ, Wu M, Engbring JA, Wang SL, Goldsmith CM, Piper JT, Vostal JG, Harms JF, Welch DR, Kleinman HK. Endogenous osteonectin/SPARC/ BM-40 expression inhibits MDA-MB-231 breast cancer cell metastasis. Cancer Res 2005; 65: 7370-7377.

25. Le Marchand L, Donlon T, Kolonel LN, Henderson BE, Wilkens LR. Estrogen metabolismrelated genes and breast cancer risk: the multiethnic cohort study. Cancer Epidem Biomar 2005; 14: 1998-2003.

26. Lesueur F, Pharoah PD, Laing S, Ahmed S, Jordan C, Smith PL, Luben R, Wareham NJ, Easton DF, Dunning AM, Ponder BAJ. Allelic association of the human homologue of the mouse modifier Ptprj with breast cancer. Hum Mol Genet 2005; 14: 2349-2356.
27. Liang YY, Hyder SM. Proliferation of endothelial and tumor epithelial cells by progestin-induced vascular endothelial growth factor from human breast cancer cells: paracrine and autocrine effects. Endocrinology 2005; 146: 3632-3641.

28. Lopez JI, Camenisch TD, Stevens MV, Sands BJ, McDonald J, Schroeder JA. CD44 attenuates metastatic invasion during breast cancer progression. Cancer Res 2005; 65: 6755-6763.

29. Luo M, Koh M, Feng JJ, Wu Q, Melamed P. Cross talk in hormonally regulated gene transcription through induction of estrogen receptor ubiquitylation. Mol Cell Biol 2005; 25: 7386-7398.

30. Mann GB, Fahey VD, Feleppa F, Buchanan MR. Reliance on hormone receptor assays of surgical specimens may compromise outcome in patients with breast cancer. J Clin Oncol 2005; 23: 5148-5154.

31. Melkoumian ZK, Peng X, Gan BY, Wu YY, Guan JL. Mechanism of cell cycle regulation by FIP200 in human breast cancer cells. Cancer Res 2005; 65: 6676-6684.

32. Oliva J, El Messaoudi S, Pellestor F, Fuentes M, Georget V, Balaguer P, Cavailles V, Vignon FO, Badia E. Involvement of HP1 alpha protein in irreversible transcriptional inactivation by antiestrogens in breast cancer cells. Febs Lett 2005; 579: 4278-4286.

33. Radvanyi L, Singh-Sandhu D, Gallichan S, Lovitt C, Pedyczak A, Mallo G, Gish K, Kwok K, Hanna W, Zubovits J, Armes J, Venter D, Hakimi J, Shortreed J, Donovan M, Parrington M, Dunn P, Oomen R, Tartaglia J, Berinstein NL. The gene associated with trichorhinophalangeal syndrome in humans is overexpressed in breast cancer. Proc Natl Acad Sci 2005; 102: 11005-11010.

34. Rouzier R, Perou CM, Symmans WF, Ibrahim N, Cristofanilli M, Anderson K, Hess KR, Stec J, Ayers M, Wagner P, Morandi P, Fan C, Rabiul I, Ross JS, Hortobagyi GN, Pusztai L. Breast cancer molecular subtypes respond differently to preoperative chemotherapy. Clin Cancer Res 2005; 11: 5678-5685.

35. Shah YM, Al-Dhaheri M, Dong Y, Ip C, Jones FE, Rowan BG. Selenium disrupts estrogen receptor alpha signaling and potentiates tamoxifen antagonism in endometrial cancer cells and tamoxifen-resistant breast cancer cells. Mol Cancer Ther 2005; 4: 1239-1249.

36. Shalom-Feuerstein R, Cooks T, Raz A, Kloog Y. Galectin-3 regulates a molecular switch from $\mathrm{N}$-Ras to K-Ras usage in human breast carcinoma cells. Cancer Res 2005; 65: 7292-7300.

37. Stassi G, Garofalo M, Zerilli M, Ricci-Vitiani L, Zanca C, Todaro M, Aragona F, Limite G, Petrella G, Condorelli G. PED mediates AKT-dependent chemoresistance in human 
breast cancer cells. Cancer Res 2005; 65: 6668-6675.

38. Sun $Y$, Strizzi L, Raafat A, Hirota M, Bianco C, Feigenbaum L, Kenney N, Wechselberger C, Callahan R, Salomon DS. Overexpression of human Cripto-1 in transgenic mice delays mammary gland development and differentiation and induces mammary tumorigenesis. Am J Pathol 2005; 167: 585-597.

39. Tu Y, Jerry DJ, Pazik B, Schneider SS. Sensitivity to DNA damage is a common component of hormone-based strategies for protection of the mammary gland. Mol Cancer Res 2005; 3: 435-442.

40. Ursin G, Bernstein L, Lord SJ, Karim R, Deapen D, Press MF, Daling JR, Norman SA, Liff JM, Marchbanks PA, Folger SG, Simon MS, Strom BL, Burkman RT, Weiss LK, Spirtas R. Reproductive factors and subtypes of breast cancer defined by hormone receptor and histology. $\mathrm{Br} J$ Cancer 2005; 93: 364-371.

41. van der Hel OL, Bueno-de-Mesquita HB, van Gils $\mathrm{CH}$, Roest M, Slothouber B, Grobbee DE, Peeters PHM. Cumulative genetic defects in carcinogen metabolism may increase breast cancer risk. Cancer Cause Contr 2005; 16: 675-681.

42. Varma AK, Brown RS, Birrane G, Ladias JAA. Structural basis for cell cycle checkpoint control by the BRCA1-CtIP complex. Biochemistry 2005; 44: 10941-10946.

43. Wang CG, Fan SJ, Li ZP, Fu MF, Rao M, Ma YX, Lisanti MP, Albanese C, Katzenellenbogen BS,
Kushner PJ, Weber B, Rosen EM, Pestell RG. Cyclin D1 antagonizes BRCA1 repression of estrogen receptor alpha activity. Cancer Res 2005; 65: 6557-6567.

44. Wittmann BM, Fujinaga K, Deng HY, Ogba N, Montano MM. The breast cell growth inhibitor, estrogen down regulated gene 1 , modulates a novel functional interaction between estrogen receptor alpha and transcriptional elongation factor cyclin T1. Oncogene 2005; 24: 5576-5588.

45. Yuan JM, Koh WP, Sun CL, Lee HP, Yu MC. Green tea intake, ACE gene polymorphism and breast cancer risk among Chinese women in Singapore. Carcinogenesis 2005; 26: 1389-1394.

46. Zhang SM, Hankinson SE, Hunter DJ, Giovannucci EL, Colditz GA, Willett WC. Folate intake and risk of breast cancer characterized by hormone receptor status. Cancer Epidem Biomar 2005; 14: 2004-2008.

47. Zhao H, Cui YZ, Dupont J, Sun H, Hennighausen L, Yakar S. Overexpression of the tumor suppressor gene phosphatase and tensin homologue partially inhibits Wnt-1-induced mammary tumorigenesis. Cancer Res 2005; 65: 6864-6873.

Prepared by R. Sutherland, J. Scorer Cancer Research Program Garvan Institute of Medical Research Darlinghurst, NSW, Australia 